\title{
Fluid flow nozzle energy harvesters
}

Stewart Sherrit, Hyeong Jae Lee, Phillip Walkemeyer, Tyler Winn, Luis Phillipe Tosi, et al.

Stewart Sherrit, Hyeong Jae Lee, Phillip Walkemeyer, Tyler Winn, Luis Phillipe Tosi, Tim Colonius, "Fluid flow nozzle energy harvesters," Proc. SPIE 9435, Sensors and Smart Structures Technologies for Civil, Mechanical, and Aerospace Systems 2015, 943507 (3 April 2015); doi: 10.1117/12.2084574 Health Monitoring, 2015, San Diego, California, United States 


\title{
Fluid flow nozzle energy harvesters
}

\author{
Stewart Sherrit ${ }^{1}$, Hyeong Jae Lee ${ }^{1}$, Phillip Walkemeyer ${ }^{1}$, Tyler Winn ${ }^{1}$, \\ Luis Phillipe Tosi ${ }^{2}$, Tim Colonius ${ }^{2}$ \\ ${ }^{1}$ Jet Propulsion Laboratory, California Institute of Technology, Pasadena, CA \\ ${ }^{2}$ Dept of Mechanical Engineering, California Institute of Technology, Pasadena, CA
}

\begin{abstract}
Power generation schemes that could be used downhole in an oil well to produce about 1 Watt average power with long-life (decades) are actively being developed. A variety of proposed energy harvesting schemes could be used to extract energy from this environment but each of these has their own limitations that limit their practical use. Since vibrating piezoelectric structures are solid state and can be driven below their fatigue limit, harvesters based on these structures are capable of operating for very long lifetimes (decades); thereby, possibly overcoming a principle limitation of existing technology based on rotating turbo-machinery. An initial survey [1] identified that spline nozzle configurations can be used to excite a vibrating piezoelectric structure in such a way as to convert the abundant flow energy into useful amounts of electrical power. This paper presents current flow energy harvesting designs and experimental results of specific spline nozzle/ bimorph design configurations which have generated suitable power per nozzle at or above well production analogous flow rates. Theoretical models for non-dimensional analysis and constitutive electromechanical model are also presented in this paper to optimize the flow harvesting system.
\end{abstract}

Keywords: Actuators, Piezoelectric Devices, Flow Energy Harvesting, bimorphs, transducers vibrators, piezoelectric power generation, fluid structure interaction

\section{Introduction}

There are several energy sources in the environment such as light, thermal, flow, and mechanical vibration that can be tapped to provide useful energy. The conversion of these ubiquitous energy sources into useful amounts of electrical energy is called energy harvesting. In this paper we are investigating using fluid flow to induce vibrations in piezoelectric transducers to generate electricity. A variety of energy harvesting systems have been developed using piezoelectric or electrostrictive materials and many distinct vibration modes have been used to generate electrical power[1,2]. The energy harvesting application we are targeting is in downhole oil/water flow with the potential for the ambient pressures to reach $30,000 \mathrm{psi}$ and a temperature up to $200{ }^{\circ} \mathrm{C}$. The necessity for energy harvesting in downhole oil producing wells is crucial, as transmitting power from the surface is complicated by difficulty of making electrical connections across production packers down inside the well. If the power can be produced locally by energy harvesting of the oil flow, the need to transmit power down the hole is removed, and downhole devices can be powered locally which reduces the overall complexity and difficulty of the system.

The approach we are investigating to harvest power is to use flow energy from the oil/water flow in downhole pipes. The mechanical energy can be converted into electrical energy through various transduction mechanisms, such as piezoelectric, electromagnetic and electrostatic[1]. Among them, piezoelectrics have been the mainstay for energy harvesting system due to their high electromechanical coupling with no external voltage source requirement $[3,4,5,6,7,8,9,10,11,12]$. In addition, they can operate with limited strain and in non rotating systems which offers the potential to produce long life harvesting systems due to limited wear; and piezoelectric materials with large piezoelectric activity and with Curie temperatures greater than $300{ }^{\circ} \mathrm{C}$ range are commercially available so the potential to operate at higher temperatures is also an advantage. A variety of studies have looked at methods to convert flow energy into vibrations including vortex shedding[13,14,15,16,17], flapping motions[18] and hydraulic pressure[19]. This paper presents the results of recent experiments on a variety of designs for flow energy harvesters which used nozzle-diffuser combinations and/or flow cavities along a pipe to produce conditions that excite a vibrating piezoelectric structure. The performance and generated average power of each nozzle investigated will be presented in this paper. 


\section{Experimental}

A schematic of the loop is shown in Figure 1. The loop has pressure gauges on the input and outputs of the energy harvester and a flow meter on the inlet to the reservoir. A safety relief valve is positioned between the pump and the accumulator. Pressure regulators are place before and after the flow energy harvester. The pump speed and flow rate are adjusted along with the inlet and outlet pressures. A filter is in between the reservoir and the pump. The system can produce up to 19 liters per min (LPM) and pump outlet pressure of 250 psi. The back pressure on the flow energy harvester (FEH) can be maintained above a critical pressure to suppress cavitation. In a newer design we are also looking at implementing digital pressure valves and a larger pump to increase the pressure up to 400 psi and flow rates of 38 LPM.

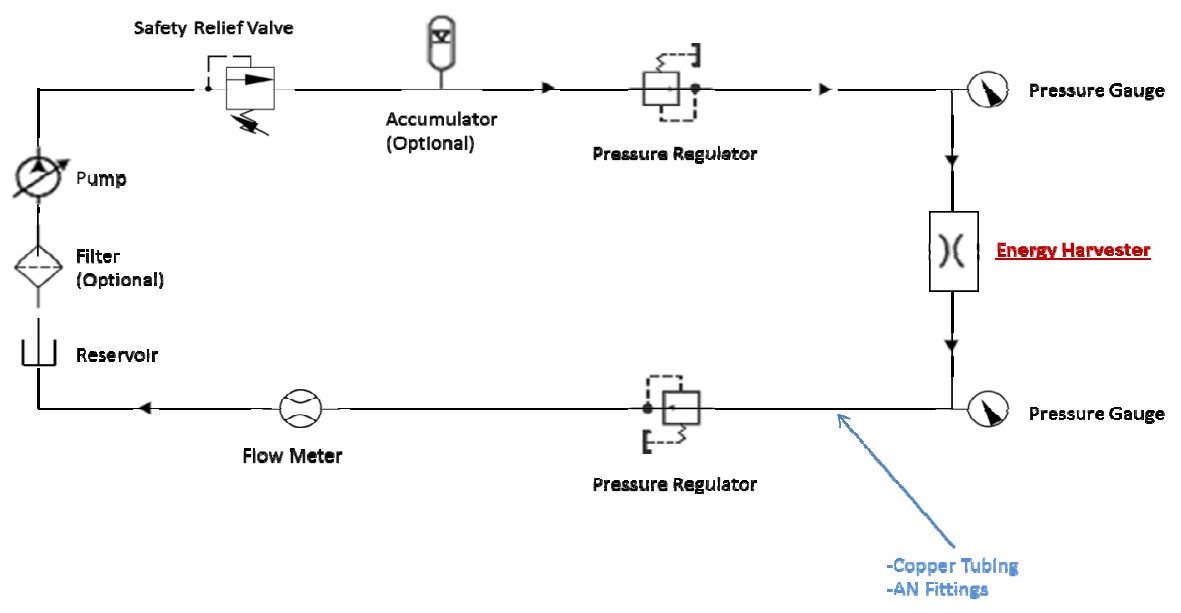

Figure 1: Schematic of the flow loop that is currently being used to measure the performance of the harvesters.

A typical flow harvester based on this design is shown in Figure 2. We have tested a variety of commercial off the shelf actuators as well as actuators with custom modifications as shown in Figure 2. The flow parameters were set in the flow loop and the flow rate, inlet pressure and outlet pressure were recorded and the load resistance across the electrical output of the harvester were measured using an Oscilloscope (TDS 2024B). The waveform V(t) vs. $t$ was downloaded to the computer and the instantaneous power $\left(\mathrm{V}(\mathrm{t})^{2} / \mathrm{R}\right)$ was time averaged to find the average DC power generated. The load resistance $\mathrm{R}$ was adjusted until a maximum in the average power was found.

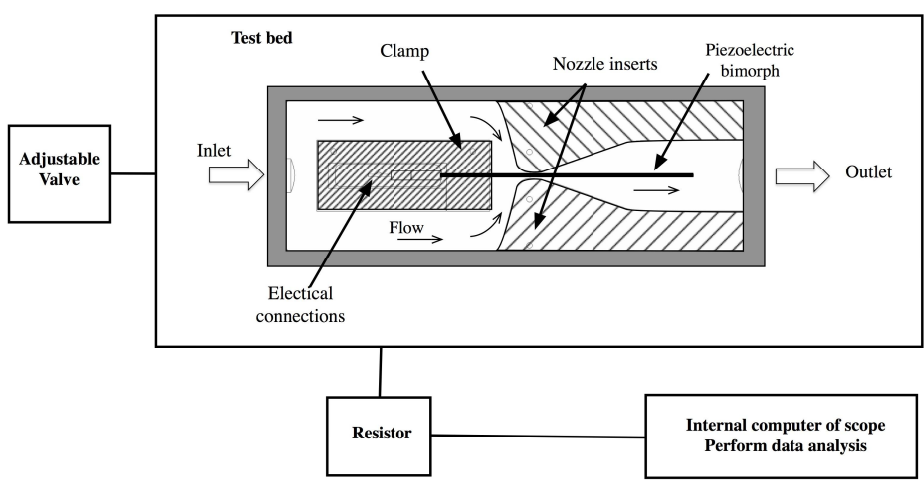

Figure 2: Schematic representation of a nominal flow energy harvester based on the spline nozzle. Flow is left to right and the nozzle profiles can be adjusted by replacing the nozzle inserts. 


\section{Performance Test Results}

\subsection{Mide V21BL harvester}

After assembling the flow loop and the experimental circuitry we investigated the standard V21BL actuators [20] in flow loop. Prior to the power measurements, electrical impedance and phase of the harvester was measured in air using an impedance analyzer in order to investigate the resonance modes of the bimorph, and the results are shown in Figure 3 (left). Note that the first resonance is found to be $140 \mathrm{~Hz}$ and the second harmonic is located at $580 \mathrm{~Hz}$.

For the measurements of the output power from this bimorph, the developed harvester system was placed inside a flow loop, then the root-mean-square (rms) voltage (Vrms) from piezoelectric bimorph was measured across a matched resistance $(2 \mathrm{kOhm})$ while increasing the flow rate from 0 to $20 \mathrm{LPM}$. The power was calculated using the relation of $V^{2} / R$, where $R$ is the resistance. The results are shown in Figure 3 (right), where the power level was increased in several steps during the tests ranged from 5 to $25 \mathrm{~mW}$, depending on flow rates.
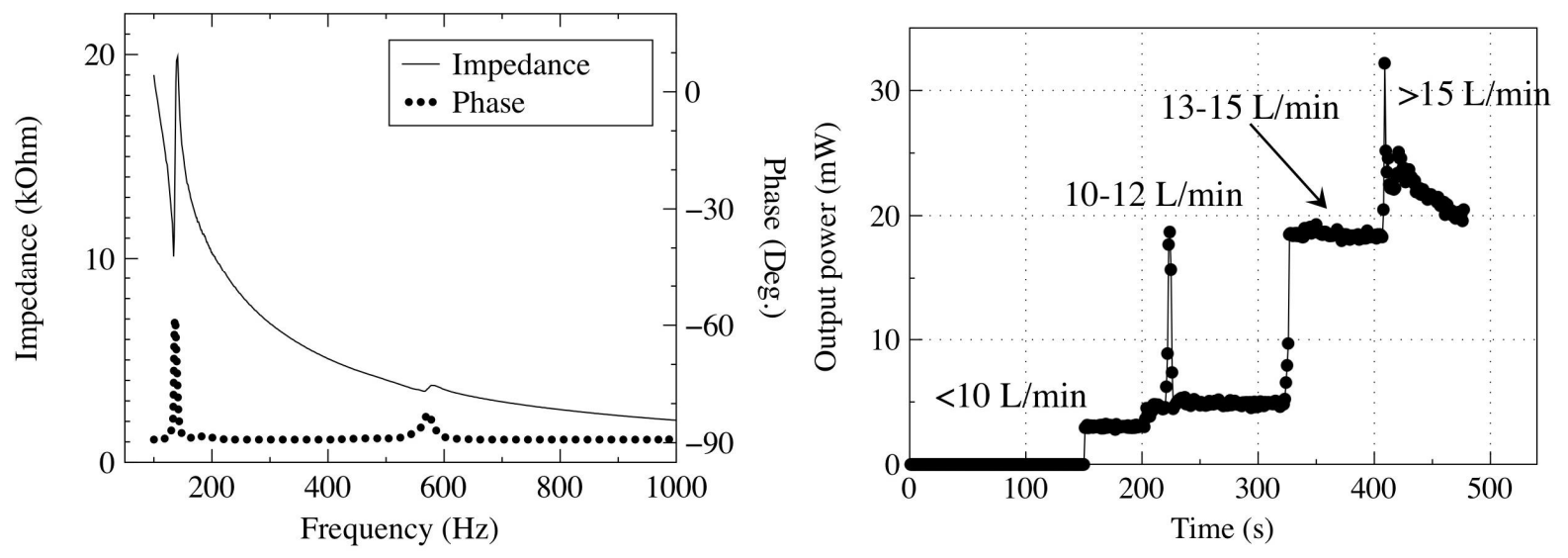

Figure 3: (solid line) Impedance and (dashed line) phase spectra of V21BL harvester as a function of frequency (left). Output power of V2IBL harvester as a function of time and flow rate (right).

Figure 4 show the measured voltage signals and fast Fourier transform (FFT) signals from V2lBL bimorph under the condition of a flow rate of 12.5 and 14 liter per $\min$ (LPM). Note that the voltage signal at a low flow rate of $12.5 \mathrm{~L} / \mathrm{min}$ showed multifrequency responses, exhibiting first $(120 \mathrm{~Hz})$ and second resonance $(450 \mathrm{~Hz})$ mode vibrations, as evident by the FFT plot. Interestingly, the vibration frequency was changed from mixed frequencies to $450 \mathrm{~Hz}$ with a small increase in flow rate from 12 GPM to 13 GPM, with a 4-5 times higher voltage output from piezoelectric bimorph. Further increase in a flow rate above $15 \mathrm{~L} / \mathrm{min}$ allows for the generation of $25 \mathrm{~mW}$ from the bimorph; however, the crack was initiated with a higher flow rate above $15 \mathrm{~L} / \mathrm{min}$, leading to a decrease in power output. 

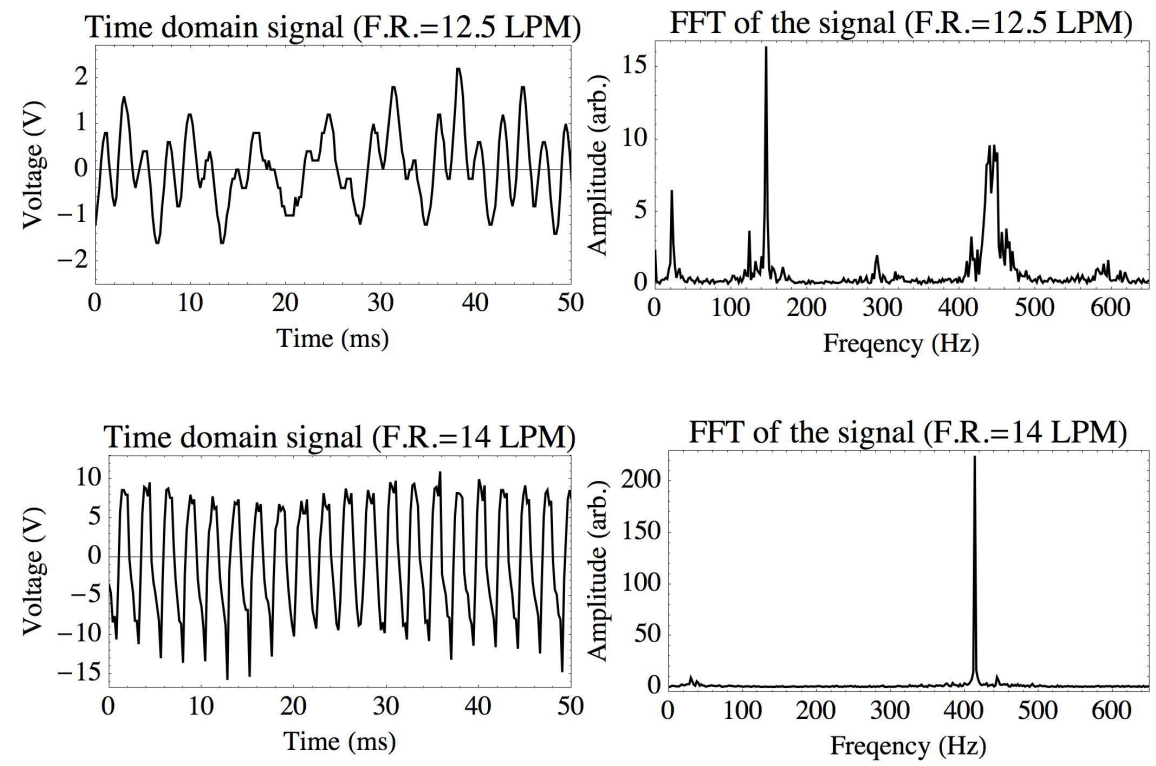

Figure 4: Time domain and fast Fourier transform of generated voltage signals from V2IBL bimorph under the condition of a flow rate of 12.5 and 14 liter per min (LPM), respectively.

Snapshots of the motion of piezoelectric bimorph under a flow rate of $14 \mathrm{~L} / \mathrm{min}$ can be found in Figure 5 . These frames were taken from a high speed camera (Nikon $1 \mathrm{~J} 4$ ) with 1200 frame per sec (fps), and showed approximately one vibration cycle of bimorph per two frames. Note that the tip displacement of bimorph was on the order of $2-3$ $\mathrm{mm}$.
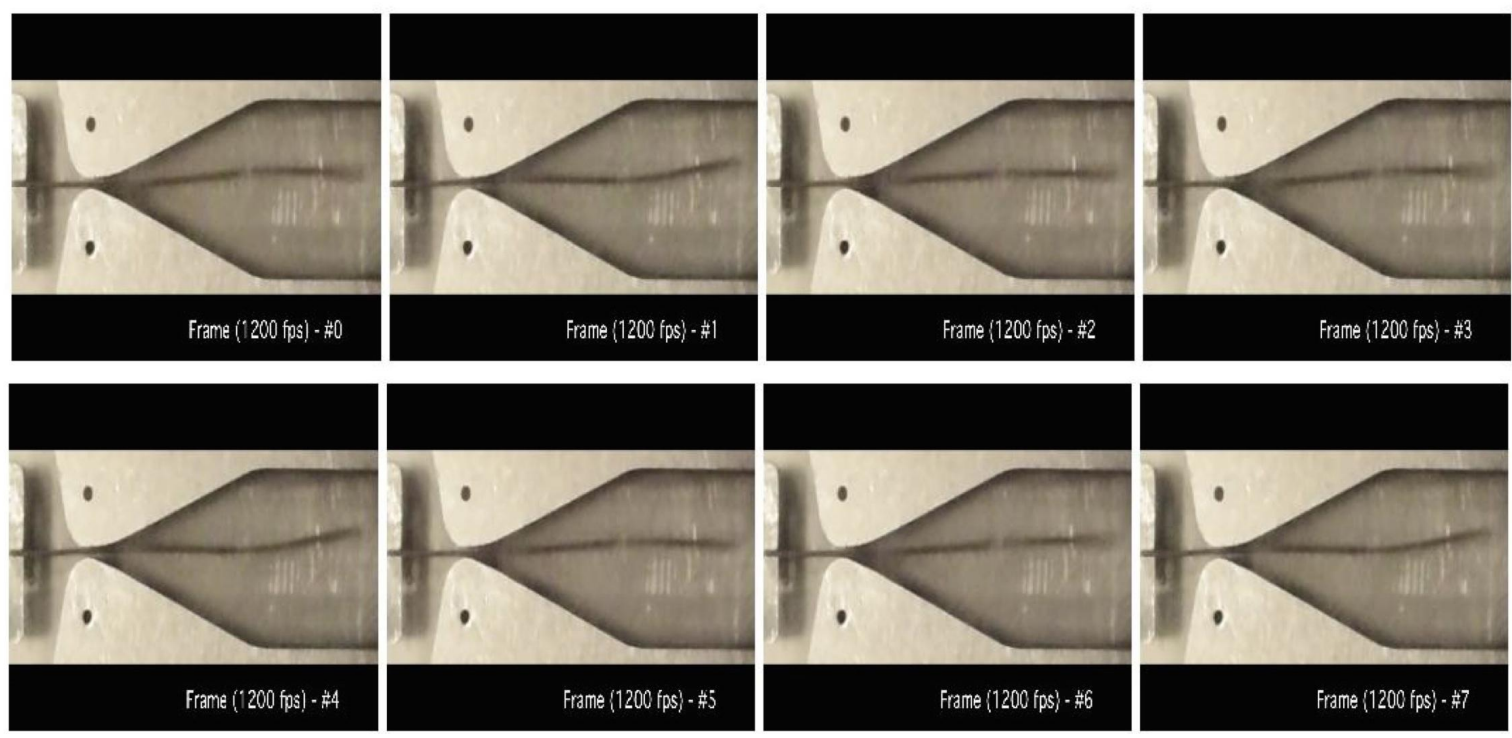

Figure 5: Snapshots of the motion of V2IBL bimorph inside flow harvester body under a flow rate of 14 $\mathrm{L} / \mathrm{min}$ using high speed camera (Frame rate $=\mathbf{1 2 0 0} \mathrm{fps}$ ).

\subsection{Metal QP21B harvester}

The Mide actuator V21BL cantilever was shown to produce up to $25 \mathrm{~mW}$ of power; however the life of the device was found to be very short and depending on the flow rate and the nozzle geometry the FEH would fail under minutes of use. This was not surprising given that we were driving these actuators well past there specified 
displacements. In order to increase the life of the actuators we investigated the ruggedization of the piezoelectric elements of the harvester by adding steel shims to the exposed surface of the cantilever. Due to thick FR4 cover layers of V21BL, QP21B actuators were selected, 2 and 5 thousands of an inch stainless steel shim were added to either side of the piezoelectric bimorph (Mide Technology Corp.). This type of bimorphs use the same piezoelectric materials (CTS - 3195HD) and dimensions as QP21B, but have thin Polymide layers instead of FR4 layers, with shorter length, compared to those of V21BL.

Photographs of the Armored (2 and 5 mil) Quick Pack (QP21B) cantilevers with steel shim are shown in Figure 6. The Quick PacK actuators shown in Figure 6 have been ruggedized for potential erosion by applying 2 and 5 thousands of an inch stainless steel shim. The electrical impedance and phase spectra of QuickPack QP21B were obtained in air and in water using impedance analyzer and the results are shown in Figure 7. Note that the resonant frequency was higher, around $400 \mathrm{~Hz}$, compared to V2lBL harvester due to the short length of the harvester. Interestingly, the first resonance frequency of QP21B in water was found to similar to that of V21BL in water, exhibiting at $140 \mathrm{~Hz}$.

The same test was performed in flow loop, and the generated output powers and exciting frequency were measured and collected, whose results are shown in Figure 7 (right). In contrast to V21BL, the power output of modified QP21B was nearly linear in terms of a flow rate, exhibiting that the output power was linearly increased to $25 \mathrm{~mW}$ with an increase in flow rates from 10 to $20 \mathrm{~L} / \mathrm{min}$. Another interesting observation is the exciting frequency was also increased with increasing the flow rate, from 140 to $170 \mathrm{~Hz}$, as shown in Figure 7 (right).

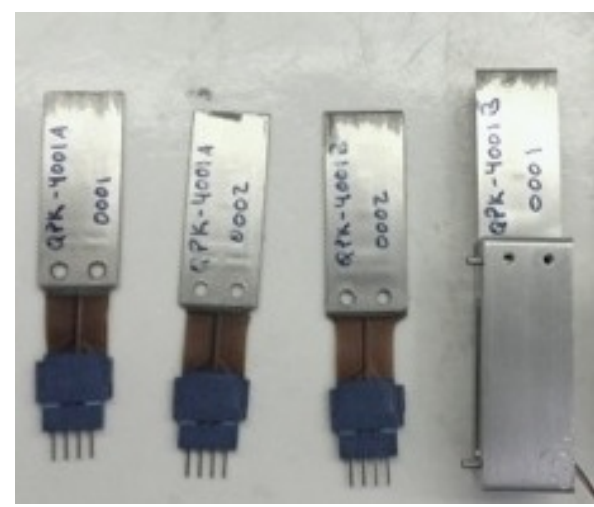

Figure 6: Photographs of the Armored ( 2 and 5 mil) QuickPack (QP21B) cantilevers with steel shim.
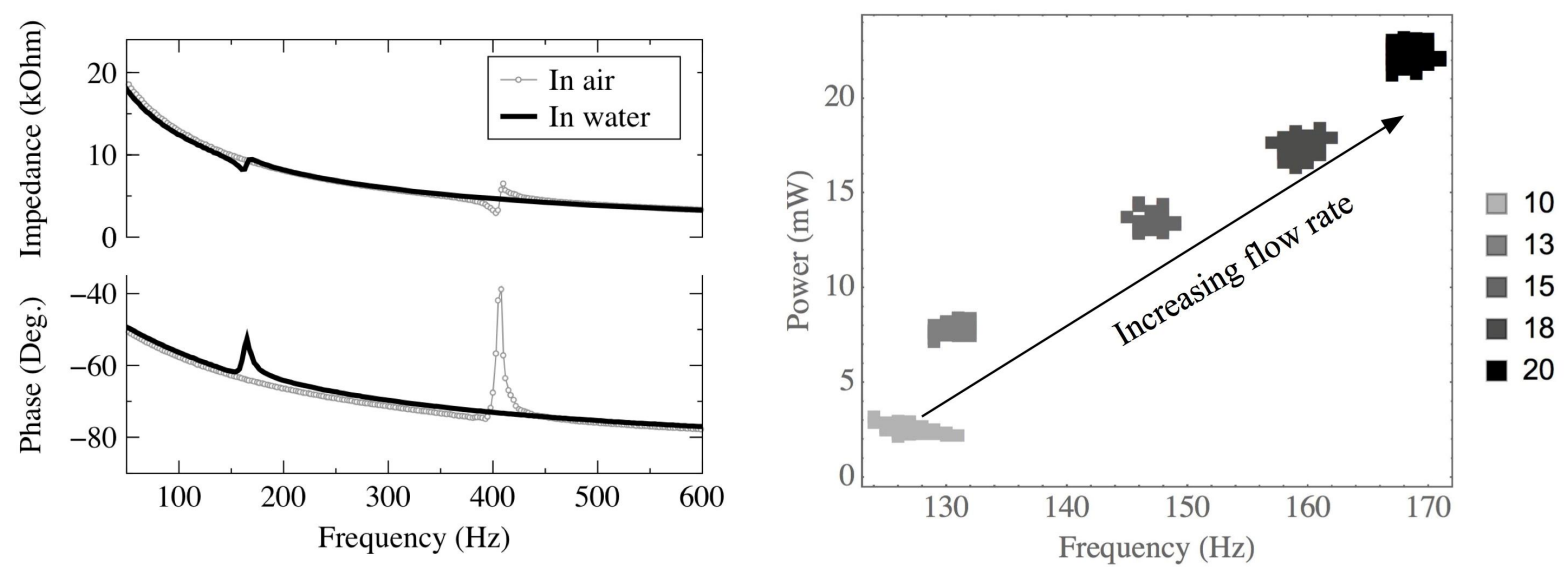

Figure 7: Electrical impedance and phase spectra of QuickPack qp21b measured in air and in water (left). Generated output power of QP21B as a function of frequency with increasing flow rate from 10 to $20 \mathrm{~L} / \mathrm{min}$. 
In order to investigate the effect on the nozzle dimensions on the power and pressure drop as a function of the flow rate, we studied a variety of nozzle dimensions with the same actuator. The results are shown in Figure 8 . The data clearly shows that the power generated has a critical flow rate at which power generation starts and then increases rapidly with increasing flow rates. The larger the gap size the larger the critical flow rate. Another trend in the data is the increase in pressure drop with decreasing gap size. It is interesting to note that at large gap size of 0.14 inches one could generate large powers at large flow rates $(15 \mathrm{~L} / \mathrm{min})$ with moderate pressure drops $(5-10 \mathrm{psi})$. The power-pressure drop data for 4 of the nozzle sizes are compiled in Figure 9.

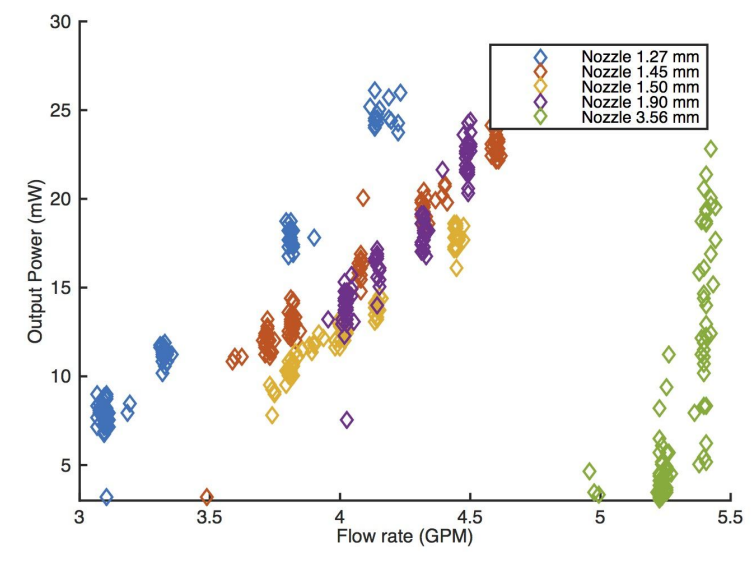

Figure 8: The power as a function of the flow rate and the pressure drop for various nozzles with gap sizes ranging from 0.05 inches to 0.14 inches.

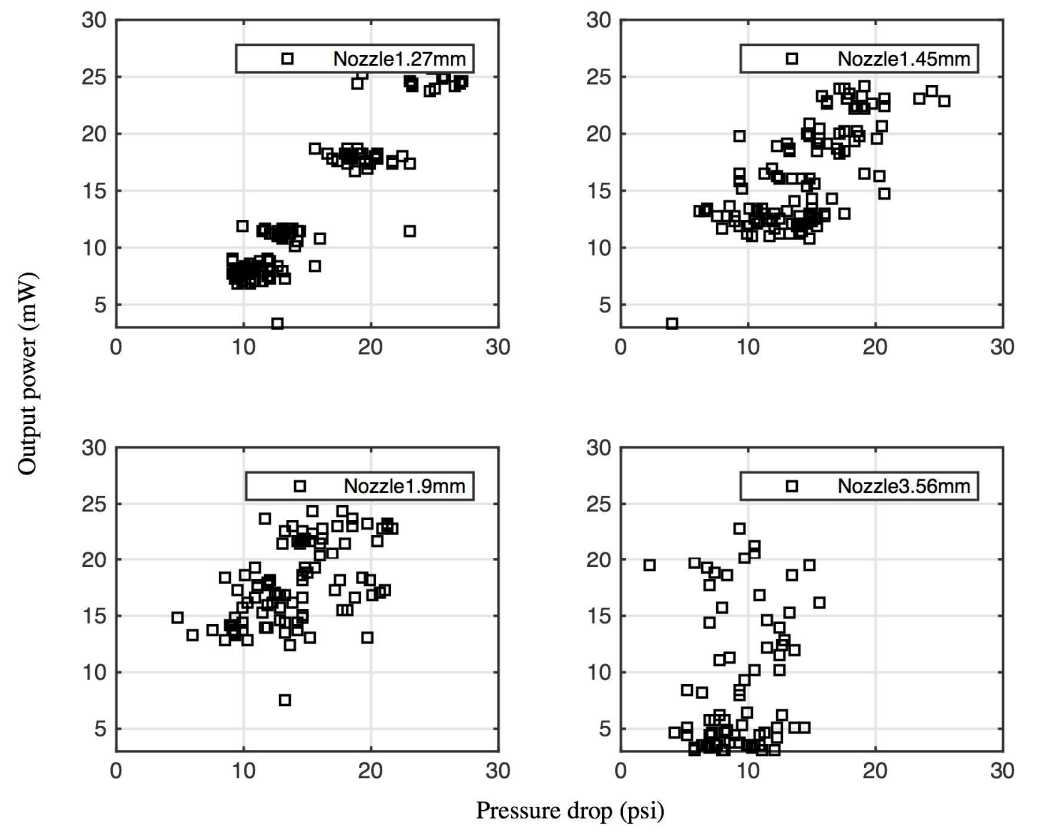

Figure 9: The power as a function of the flow rate and the pressure drop for various nozzles with gap sizes ranging from 0.05 inches to 0.14 inches. 


\subsection{Video Data Power Analysis}

An alternate means of calculating power output and a first order approximation of the stress profile on different layers of the bimorph actuator used the video data to map the beam shape into power extracted. Although the method needs much refining, this section aims at discussing the details of the method and preliminary results. The initial analysis takes the cantilever as a forced Euler-Bernoulli beam with clamped- and free- end boundary conditions. This implies that bending stresses are dominant over shear stresses, and that small strain and small beam displacement relative to the beam length $L$ exist. The shape of the beam was approximated by an eigenfunction expansion that satisfies the above boundary conditions for the eigenvalue problem of the biharmonic operator [21]:

$$
\phi_{n}(x)=\left(\cosh \left(\beta_{n} x\right)-\cos \left(\beta_{n} x\right)+a_{1} \sin \left(\beta_{n} x\right)-a_{2} \sinh \left(\beta_{n} x\right)\right)
$$

where the wave number $\beta$ satisfies

$$
\cos \left(\beta_{n} L\right) \cosh \left(\beta_{n} L\right)=-1
$$

and the constants

$$
\begin{aligned}
& a_{1}=\frac{\cos \left(\beta_{n} L\right)+\cosh \left(\beta_{n} L\right)}{\sin \left(\beta_{n} L\right)+\sinh \left(\beta_{n} L\right)} \\
& a_{2}=\frac{\cos \left(\beta_{n} L\right)+\cosh \left(\beta_{n} L\right)}{\sin \left(\beta_{n} L\right)+\sinh \left(\beta_{n} L\right)}
\end{aligned}
$$

with $x$ as the coordinate along the beam length. The displacement $\delta$ is

$$
\delta(x, t)=\sum_{n=1}^{\infty} \xi_{n}(t) \phi_{n}(x)
$$

where $\xi_{n}(t)$ is a periodic function of time.

Figure shows the beam deflection $\delta$ as a function of $x$ and time $t$.

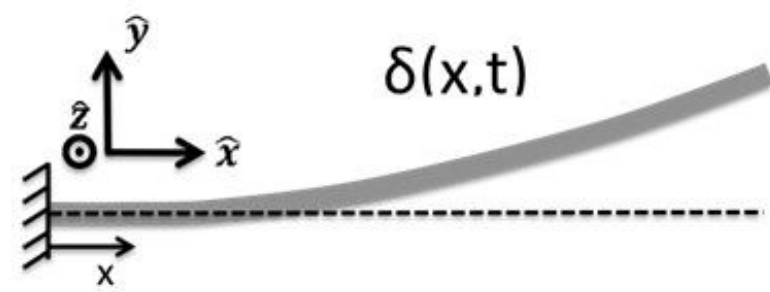

Figure 10: illustration of deformed beam represented by $\delta(x, t)$ with coordinate system.

Slow motion camera data was processed frame by frame and the edges of the vibrating cantilever were mapped in the $x-y$ plane, shown in Figure, using the canny filter implementation in MATLAB [22]. The filter parameters were chosen as necessary to consistently capture the edges near the same location $( \pm 1$ pixel) for each video file. The actuator experiments chosen for the initial processing consisted of vibration almost entirely in their fundamental mode. Hence, only the $n=1$ solution to equation (2) is considered for the cases shown. At each frame, the $x-y$ edge data is least squares fitted to equation (1), yielding a constant coefficient for $\xi_{1}\left(t_{i}\right)$, where index $i$ is the frame number. $\xi_{1}(t)$ is a sinusoid fit to the time series $\xi_{1}\left(t_{i}\right)$ with the appropriate frequency and phase parameters. 
As shown by Sodano, et. al [23], to calculate the power output from $\delta(x, t)$, the piezoelectric constituent equations applied to the bimorph actuator geometry yields a first order ODE for the charge $q(t)$ as

$$
\boldsymbol{R} \dot{q}(t)-C_{p}^{-1} \Theta_{i} \xi_{i}(t)+C_{p}^{-1} q(t)=0
$$

where $R$ is the circuit resistance and the dot represents the derivative in time. The capacitance is

$$
C_{p}=\iiint_{V_{p}} \psi^{2}(y) \varepsilon d x d y d z
$$

with $V_{p}$ as the bounds to the piezoelectric volume. The electromechanical coupling constant is

$$
\Theta_{1}=-\iiint_{V_{p}} y e \phi_{1}^{\prime \prime}(x) \psi(y) d x d y d z
$$

where $e$ is the piezoelectric coupling coefficient and $y$ is the coordinate variable, and the function

$$
\psi(y)=\left\{\begin{array}{cc}
-\frac{1}{t_{p}} & \frac{t}{2}<y<\frac{t}{2}+t_{p} \\
0 & -\frac{t}{2}<y<\frac{t}{2} \\
\frac{1}{t_{p}} & -\frac{t}{2}-t_{p}<y<-\frac{t}{2}
\end{array}\right.
$$

for $y$ values shown in Figure .
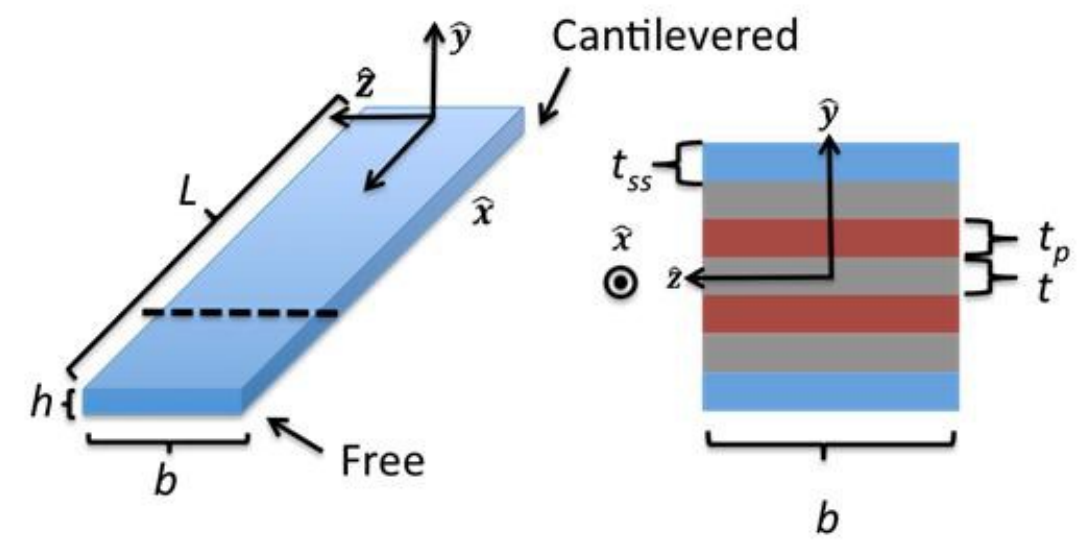

Figure 11: Illustration of bimorph cantilevered and cross-section. Red represents the piezoelectric material layer, grey the polyimide coating, and blue the 301 stainless steel layer for the geometry of the armored bimorph.

Using variation of parameters, the steady state solution to equation (6) is

$$
q_{s s}(t)=\int_{0}^{t} \exp \left[\left(R C_{p}\right)^{-1}(t-\tau)\right]\left(R C_{p}\right)^{-1} \Theta_{1} \xi_{1}(\tau) d \tau
$$

and the instantaneous power output $P$ 


$$
P(t)=\dot{q}_{s s}^{2} R
$$

Video for the armored QuickPack (QP21B) was analyzed for the experiments with a nozzle throat size of $1.27 \mathrm{~mm}$ and flow rates of $2.50 \mathrm{gal} / \mathrm{min}, 3.26 \mathrm{gal} / \mathrm{min}, 4.02 \mathrm{gal} / \mathrm{min}$, and $4.70 \mathrm{gal} / \mathrm{min}$ shown in plot . The piezoelectric coupling coefficient $e=1.5052 \mathrm{E}-08 \mathrm{~m} / \mathrm{V}$ and resistance $R=10 \mathrm{kOhms}$. Figure 12 shows the results of the analysis, plotting the predicted power output with an error bound of \pm 1 pixel and the time average of the root mean square (RMS) oscilloscope measured power.

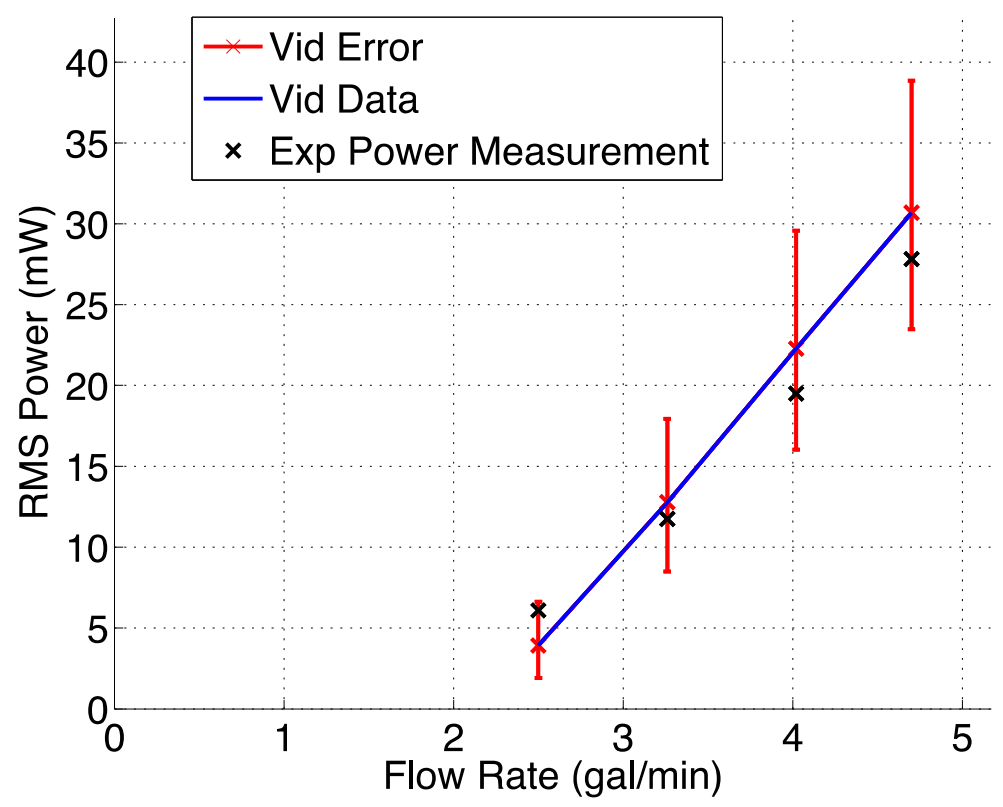

Figure 12: video processed power data (blue and red lines) and oscilloscope measurement (black $x$ )

Although the model is limited to assumptions of the Euler-Bernoulli beam and the boundary conditions mentioned, it seems to do a reasonable job at predicting the RMS power measured.

\subsection{Long Term Stability Test Results}

Testing at the larger flow rates and tip displacements found that fatigue in these layers was a problem and the initial samples lasted less than one hour and the stainless still shim and piezoelectric layers were found to fatigue and break. In the first test the parameters were: flow rate $=4.38 \mathrm{GPM}$, vibration freq. $=$ $155 \mathrm{kHz}$, resistance $=10 \mathrm{kOhm}$, inlet pressure $=75 \mathrm{psi}$, outlet pressure $=35$ psi. The results are shown in Figure 13 as a function of time. Note that as can be seen from the plot, the power is moderately flat up to about 40 minutes; however, after $40 \mathrm{~min}$, the power was dropped off to zero, showing permanent damage on the bimorphs. The photographs of the Mide actuators after testing are shown in the small inset of Figure 13, suggesting that the cause of the failure was due to the fact that the actuators were being driven past their fatigue limits. 


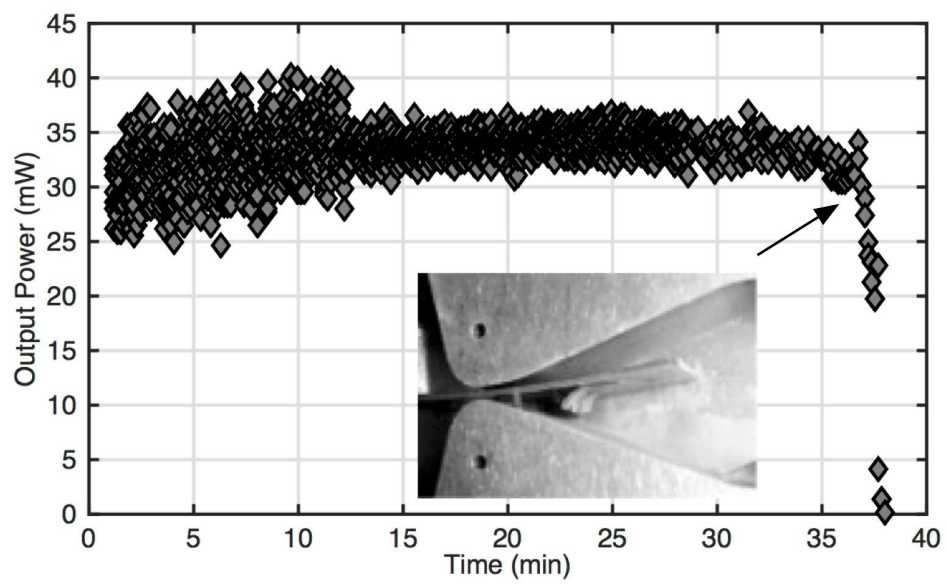

Figure 13: The output power of QP21B as a function of the time (small insets show photographs of the fatigued actuators after testing).

Another test was initiated with lower flow rates and lower tip displacements, and the results are shown in Figure 14. The test parameters were: flow rate $=2.4 \mathrm{GPM}$, vibration freq. $=120 \mathrm{kHz}$, resistance $=10 \mathrm{kOhm}$, inlet pressure $=22 \mathrm{psi}$, outlet pressure $=7 \mathrm{psi}$. The tip displacement and the power generated were reduced for this test as were the inlet and outlet pressures. The test was run for 9 hours ( $3,888,000$ cycles), and the harvester did not show any physical damages and power degradation during 9 hours; however after 9 hours a crack in the metal shim where the actuator was mechanically fixed developed. The test was stopped and the actuator examined. The results suggest that the rigid fixture clamping the bimorph may have been responsible for the fatigue and the joint. We are currently looking at methods to reduce the stress at this joint. The initial results suggest the damage due to delamination and bending strain in the actuator near the nozzle throat have been mitigated; however, we now have a critical stress and the mounting point.

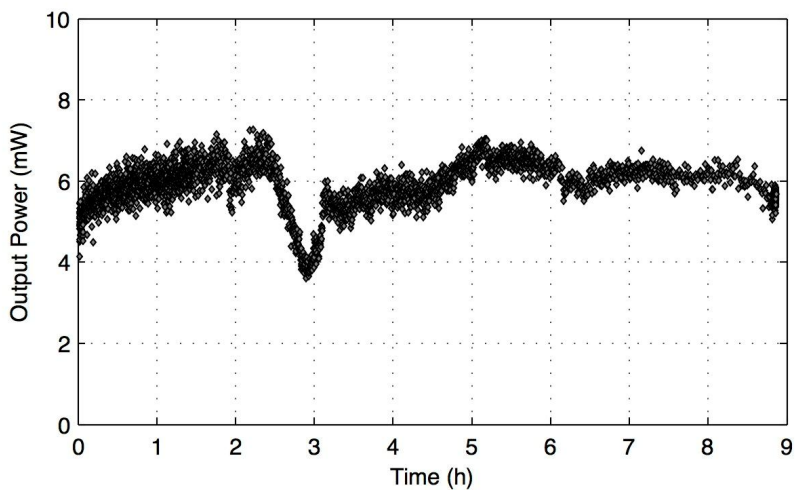

Figure 14: The output power of QP21B harvester as a function of the time with low tip displacement and flow rates.

\section{Conclusion and Future work}

Flow energy piezoelectric harvesters were investigated, and it was found that spline nozzle design showed superior performance over other nozzle designs, generating upto $30 \mathrm{~mW}$ power at a matched resistance. It was noted however, that under extended use the bimorph was damaged due to impacts of the bimorph on the throat of the spline nozzle, and its performance decreased. In addition when driven at high flow 
rates, the strain in the bimorph exceeded the ultimate strain and cracks formed causing a reduction in life as well as a power degradation. The fatigue in these designs reduced the life of the actuators to less than 40 minutes. The lifetime was increased to over 9 hours by reducing the flow rate and the power generation to about $6 \mathrm{~mW}$ average power. In order to solve this issue, new transducer designs are currently being investigated, where we have mounting structures that reduce the stress and the flow rate is adjusted to drive the cantilever below the suggested tip displacement. An analysis of the failure mechanism due to fatigue is currently being explored using detailed analysis of the video data available. In addition to the lifetests we also looked and the parameterization of the power, pressure drop and flow rates for a 5 different nozzle throat sizes. This allows for ability to begin a system design to produce the desired system power.

\section{ACKNOWLEDGMENT}

The research at the Jet Propulsion Laboratory (JPL), a division of the California Institute of Technology, was carried out under a contract with the National Aeronautics Space Agency (NASA). Reference herein to any specific commercial product, process, or service by trade name, trademark, manufacturer, or otherwise, does not constitute or imply its endorsement by the United States Government or the Jet Propulsion Laboratory, California Institute of Technology.

\section{REFERENCES}

[1] S. Sherrit, "The physical acoustics of energy harvesting," Proceedings of the IEEE Ultrasonics Symposium -IUS 2008, 1046-1055, (2008)

[2] Sherrit, S., Lee, H. J., Walkemeyer, P., Hasenoehrl, J., Hall, J. L., Colonius, T., ... \& Corbett, G. (2014, April). Flow energy piezoelectric bimorph nozzle harvester. In SPIE Smart Structures and Materials + Nondestructive Evaluation and Health Monitoring (pp. 90570D-90570D). International Society for Optics and Photonics.

[3] S. Priya, "Advances in energy harvesting using low profile piezoelectric transducers," Journal of Electroceramics, vol. 19, no. 1, pp. 167-184, (2007)

[4] N. S. Shenck, "A demonstration of useful electric energy generation from piezoceramics in a shoe," Ph.D. dissertation, Massachusetts Institute of Technology, Dept. of Electrical Engineering and Computer Science, (1999)

[5] J. A. Paradiso and T. Starner, "Energy scavenging for mobile and wireless electronics," Pervasive Computing, IEEE, vol. 4, no. 1, pp. 18-27, (2005)

[6] S. R. Platt, S. Farritor, and H. Haider, "On low-frequency electric power generation with pzt ceramics," Mechatronics, IEEE/ASME Transactions on, vol. 10, no. 2, pp. 240-252, (2005)

[7] M. Lallart, L. Garbuio, L. Petit, C. Richard, and D. Guyomar, "Double synchronized switch harvesting (dssh): a new energy harvesting scheme for efficient energy extraction," Ultrasonics, Ferroelectrics and Frequency Control, IEEE Transactions on, vol. 55, no. 10, pp. 2119-2130, (2008)

[8] N. S. Shenck and J. A. Paradiso, "Energy scavenging with shoe-mounted piezoelectrics," Micro, IEEE, vol. 21no. 3, 30-42, (2001)

[9] D. Guyomar, A. Badel, E. Lefeuvre, and C. Richard, "Toward energy harvesting using active materials and conversion improvement by nonlinear processing,", IEEE Transactions on Ultrasonics, Ferroelectrics and Frequency Control, vol. 52-no. 4, 584-595, (2005) 
[10] A. Soma and G. De Pasquale, "Design of high-efficiency vibration energy harvesters and experimental functional tests for improving bandwidth and tunability," SPIE Microtechnologies, U. Schmid, J. L. S' anchez de Rojas Aldavero, and M. Leester-Schaedel, Eds. SPIE, 87630U., (2013)

[11] J. Dias, C. De Marqui, and A. Erturk, "Electroaeroelastic modeling and analysis of a hybrid piezoelectricinductive flow energy harvester," Proceedings of SPIE Smart Structures and Materials + Nondestructive Evaluation and Health Monitoring. International Society for Optics and Photonics, 86 882N-86 882N, (2013)

[12] D. Zhu, Vibration Energy Harvesting: Machinery Vibration, Human Movement and Flow Induced Vibration, (2011)

[13] J. J. Allen and A. J. Smits, "Energy Harvesting Eel,” Journal of Fluids and Structures, vol. 15- no. 3-4, 629640, (2001)

[14] E. Bischur, S. Pobering, M. Menacher, and N. Schwesinger, "Piezoelectric energy harvester operating in flowing water," Proc. of SPIE , vol. 7643, 76 432Z-1, ( 2010)

[15] X. Gao, W.-H. Shih, and W. Y. Shih, "Flow Energy Harvesting Using Piezoelectric Cantilevers With Cylindrical Extension," IEEE Transactions on Industrial Electronics, vol. 60- no. 3, 1116-1118, (2013)

[16] E. Molino-Minero-Re, M. Carbonell-Ventura, C. Fisac-Fuentes, A. Manuel-Lazaro, and D. M. Toma, "Piezoelectric energy harvesting from induced vortex in water flow," Proceedings of the IEEE International Instrumentation and Measurement Technology Conference (I2MTC), 624-627, (2012)

[17] J. D. Hobeck and D. J. Inman, "Electromechanical and statistical modeling of turbulence-induced vibration for energy harvesting," Proceedings of the SPIE Smart Structures and Materials+ Nondestructive Evaluation and Health Monitoring , 86 881P, (2013)

[18] M. Bryant, M. W. Shafer, and E. Garcia, "Power and efficiency analysis of a flapping wing wind energy harvester", Proceedings of the SPIE Smart Structures and Materials+ Nondestructive Evaluation and Health Monitoring. 83410E, (2012)

[19] E. A. Skow, K. A. Cunefare, and A. Erturk, "Design and performance enhancement of hydraulic pressure energy harvesting systems," Proceedings of the SPIE Smart Structures and Materials+ Nondestructive Evaluation and Health Monitoring, 868803, (2013)

[20] Mide Technology Corp. Available: http://www.mide.com

[21] D. J. Inman, “Engineering Vibration”, New Jersey: Pearson, (2008)

[22] J. Canny, "A Computational Approach to Edge Detection," IEEE Transactions on Pattern Analysis and Machine Intelligence, Vol. PAMI-8, No. 6, 679-698, (1986)

[23] H. A. Sodano, G. Park, and D. J. Inman. "Estimation of electric charge output for piezoelectric energy harvesting." Strain 40.2, 49-58, (2004) 